\title{
Management and Business of Autonomous Vehicles: A Systematic Integrative Bibliographic Review
}

Bruna Habib Cavazza ${ }^{1,2}$, Rodrigo Marçal Gandia ${ }^{1}$, Fabio Antoniallii, ${ }^{1,2}$, Isabelle Nicolaï ${ }^{2}$, André Luiz Zambalde ${ }^{1}$, Joel Yutaka Sugano ${ }^{1}$, Arthur de Miranda Neto ${ }^{1}$

${ }^{1}$ Laboratório de Mobilidade Terrestre - Universidade Federal de Lavras, Lavras, Brazil.

${ }^{2}$ Laboratorie Genie Industriel - CentraleSupélec / Université Paris-Saclay, Châtenay-Malabry, France.

brunacavazza@gmail.com

romgandia@gmail.com

fantonialli@gmail.com

isabelle.nicolai@centralesupelec.fr

zamba@dcc.ufla.br

joel.sugano@dae.ufla.br

arthur.miranda@deg.ufla.br

Abstract: This paper aims at characterizing the Autonomous Vehicles (AVs) research field in the areas of management and business in its bibliometric context; identifying strategies, practices and management tools specified in the scope of the investigated publications; summarizing existing evidence, pointing to gaps within this study area. Methodologically, the research is characterized as qualitative and descriptive, drawn by a bibliometric review on the databases; ISI Web of Science, Scopus and Science Direct, followed by a systematic integrative bibliographic review. All the titles and abstracts of the identified articles were analyzed allowing for a research refinement, adopting the exclusion criteria for; a) duplicates; b) not obtained references; and c) misaligned references. The main results pointed out that, in the near future AVs will certainly be inserted in our society, however the way in which this innovation might be established is still surrounded by uncertainties, impacting directly on governments' lack of planning for such arrival (Guerra, 2016). The absence of work related to the business area can be a driving factor, considering that business models plays an extremely important role in the events that precede the AVs' advancement (Yun et al., 2016). Nevertheless, among the analyzed papers, a studies' trend is highlighted, especially in European countries (e. g. U.K. and Germany), related to AVs' business model of "car-sharing" (Zakharenko, 2016; Geldmacher, 2016); presenting such as a great substitute for traditional transportation models (cars, taxis and buses) (Enoch, 2015). In this way, it was observed a study gap related to business models and platforms, radical and responsible innovation theories, in order to minimize the risks, impacts and uncertainties of the eminent arrival of $A V s$ and provide the necessary tools to guide governmental and organizational spheres.

Keywords: Autonomous Vehicles, Business, Management, Systematic Integrative Review, Bibliometric Review

\section{Introduction}

The development of Autonomous Vehicles (AVs) is an important innovation that promises to have great impact on the issues of urban mobility. In fact, AVs are embedded in the most significant historical change to the automobile and transportation industry. Governments and universities worldwide identify AVs as keyresearch factor. The AVs' imminent arrival includes impacts on several spheres, being important to consider the impacts of such disruptive innovation on society, structure and functioning of companies. Among the stakeholders responsible for the dissemination of this innovation, the academy is understood as an important precursor on AVs' development, being responsible for important theoretical and empirical research for the field's evolution. Although technological advances are essential for this innovation dissemination, it is understood that, in the case of radical innovations, technology cannot be established as the process' exclusive key-factor, but combined with the establishment of a business model that supports it (Christensen et al., 2006).

Notwithstanding, the advancement of technological innovations that permeate AVs and the intelligent robot industry is part of a dynamic result established among technology, business models, and marketplace (Yun et al., 2016). In this context, the possibility of changes in the dynamic relations among these three factors in order to obtain expected results, is on evidence. Although there are some studies that seek to make a general analysis of the context and environment of $A V s$, there is a lack of theoretical and empirical studies seeking to analyze AVs from a market perspective, related to aspects of planning and management. Thus, it is important to look at the literature approaches related to management and business (M\&B) of AVs; attempting to know and spread a little more about the reality of these issues, by Interpreting and integrating content, summarizing existing evidence, identifying key issues and finding research gaps.

Considering the aforementioned, this study aims at answering the following research question: " How does the AVs field of study relate to the management and business (M\&B) area?" Specifically, it aims at performing 
an integrative bibliometric and systematic review of the literature focusing on investigating and understanding the main theoretical contributions related to AVs in the M\&B field of study.

Therefore, this study is justified because - characterizing the AVs research field in the M\&B areas in its bibliometric context; identifying strategies, practices and management tools specified in the scope of the investigated publications; summarizing existing evidence, pointing to little or no explored topics (gaps) within this area of study - will contribute to the field' systematization based on evidence pointed out in the literature.

In this context, a bibliometric and systematic review is important on allowing us to focus specifically on the subject, identify authors, articles, dates, journals and approaches; review the primary studies; integrate and synthesize the evidence associated with results involving practical strategies, applications and tools and finally, work and disseminate specific information in a chronologically organized and directed way (Botelho; Cunha \& Macedo, 2011).

\section{Autonomous Vehicles overview}

AVs, which are vehicles that don't require any sort of conductor or teleoperation control are of great interest and investment in the global automotive industry with numerous carmakers already testing AVs' technologies (Frazzoli, Dahleh \& Feron, 2002; Fagnant \& Kockelman, 2015). AVs are also relevant to governments in a sense that many countries are changing legislations to allow testing of such vehicles on their roads (Schoitsch, 2016), as well as are relevant to the academia with research centers and universities worldwide advancing many studies on technology mobility, vehicle-infrastructure interaction and M\&B related issues for AVs' consolidation (Lima, 2015).

Thus, AVs represent a potentially disruptive and beneficial change to the transportation system business model, since they will facilitate driving; increase road safety; reduce pollutants' emissions; reduce traffic jams; as well as allow drivers to choose to do different things other than driving. AVs will also improve mobility for those who cannot or do not want to drive and will provide significant economic, environmental and social benefits (Attias, 2016; Mutz et al., 2016).

\section{Bibliometric and systematic review}

Literature review articles are "a form of research using sources of bibliographic or electronic information to obtain search results from other authors, in order to theoretically support a particular topic" (Botelho; Cunha \& Macedo, p.124, 2011).

In bibliometric reviews, we have the quantification of publications related to a topic under study, that is, frequency of publications over the years, authors, journals, countries, and other important data. On the other hand, systematic literature reviews should be designed to address a particular issue with the use of detailed, explicit and systematic methods to raise, identify, select, interpret, collect and analyze references (Botelho; Cunha and Macedo, 2011).

According to Whitemor \& Knafl (2005) there are basically four types of systematic literature review: metaanalysis (a combination of results of studies with statistical formulas targeting analysis, comparisons and generate new results); systematic (is based on scientific research strategies, limits the scope of selection of articles, synthesizes studies and evaluates them critically); qualitative (systematization and synthesis of qualitative studies' findings transforming them into tools to build new theories) and integrative (reviews the past empirical and theoretical literature, presenting the state of the art on a theme, especially for results analysis and synthesis of previous research contributions and gaps).

In this study, we performed a bibliometric review followed by an integrative systematic literature review. The integrative review term refers to the fact that in this method, concepts, opinions and ideas arising from previous research are integrated (Whitemore \& Knafl, 2005).

\section{Methodology}

The research is characterized as qualitative and descriptive, drawn by a bibliometric review and by a systematic integrative bibliographic review focusing on investigating and understanding the main theoretical contributions related AVs in the M\&B field of study, Figure 1 highlights the research design. 


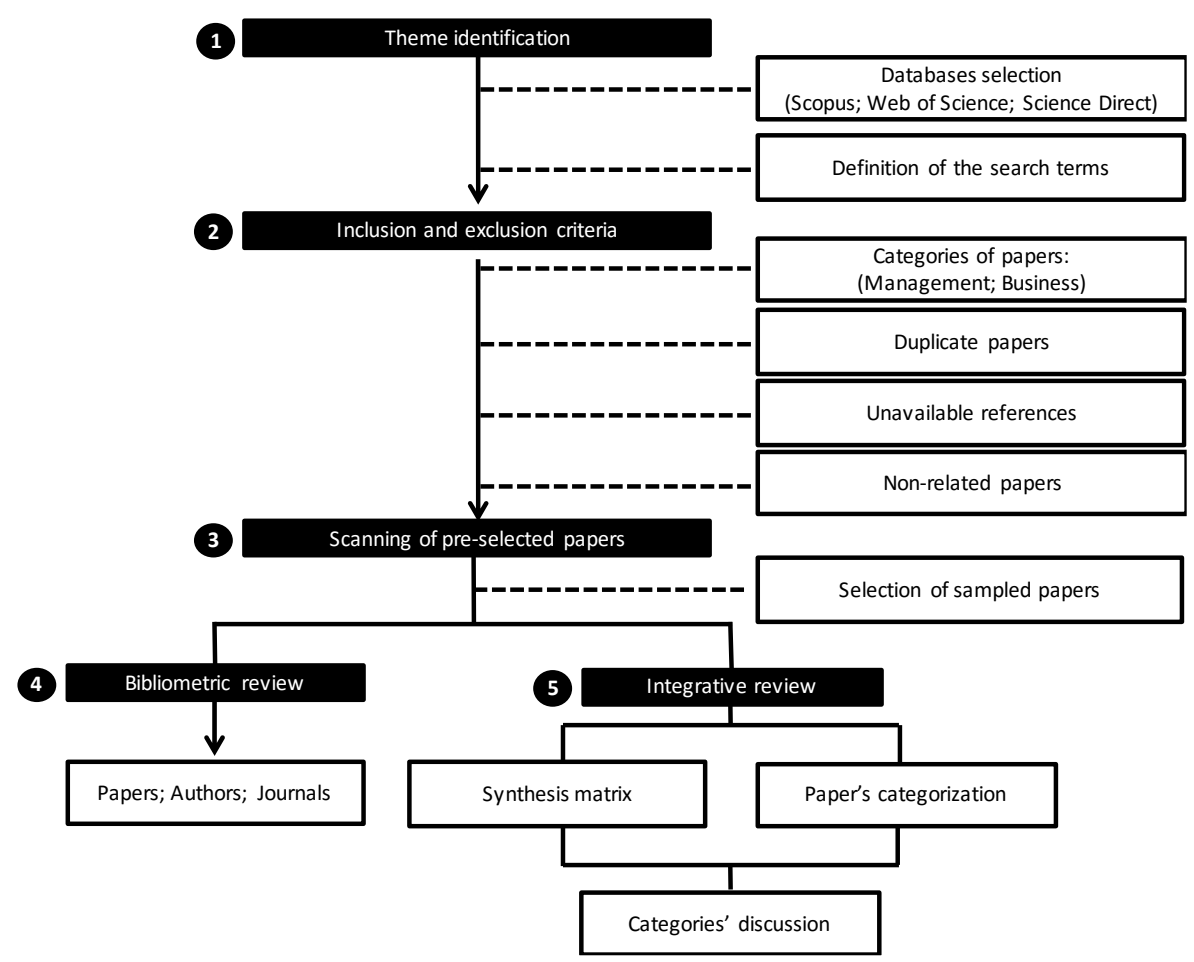

Figure 1: Research design.

- Theme identification (step 1): the articles were searched on Scopus; Web of Science (WoS) \& Science Direct (SD) databases in a single search, between 1945 to 2017, using the Boolean operator "OR" and were selected by the following terms in the title, abstract and keywords of the articles: autonomous_car*; autonomous_vehicle*; autonomous_automobile*; driverless_car*; driverless_vehicle*; driverless_automobile*; self-driving_car*; selfdriving_vehicle*; self-driving_automobile*; intelligent_car*; intelligent_vehicle* and intelligent_automobile*. We found 6,711 papers from WoS; 27,855 papers from Scopus and 6,796 papers from SD. It is worth clarifying that the Boolean operator underline '_' was used to ensure that the search yielded results in which only the pair of words appeared together. The operators star '*' (WoS and Scopus) and "' (SD) were used to ensure that both singular and plural terms were included in the search results.

- Inclusion and exclusion criteria (step 2): the first criterion was to select publications classified as articles within the databases' M\&B categories. We found 644 papers: 244 from Scopus, 56 from WoS and 344 from SD. All articles' titles and abstracts were analyzed in order to allow further filtering. Of the 644 articles, 613 were excluded following these criteria: a) duplicate references - identified more than once in different sources (07 papers); b) unachievable references - that could not be obtained by interlibrary loan, online search or contacting the authors (03 papers) and; c) non-related references to the research problem or just tangent the themes of interest (604 papers).

- Scanning of pre-selected papers (step 3): after step 2, we reached the number of 30 papers for research and analysis. Those papers were pre-selected for in-depth reading and analysis.

- Bibliometric review (step 4): in this step, we analyzed the evolution of publications over the years, countries frequency' publications, publications' languages, methodological approach of the papers, most cited paper and also key-words recurrence in a Word Cloud.

- Integrative review (step 5): initially we carried out a detailed study of the main issues of each document (synthesis matrix), including: key words; research problem; goals; theoretical background; study type; methodological approach; main contributions; future research proposals. After, their appropriate categorization within themes, facilitating the presentation and discussion of results. It is worth mentioning that this categorization was performed by two members of the team and later validated by a third person in order to guarantee impersonality in the results.

\section{Results and discussion}

\subsection{Bibliometric review}


The first analysis we present regards the number of publications per year. Figure 2 displays the distribution of the 30 articles found in accordance with the year of publication. It is observed that the first publication is the 1990 - article "Intelligent Vehicle-Highway Systems: U.S. activities and policy issues" (Chen \& Ervin, 1990).

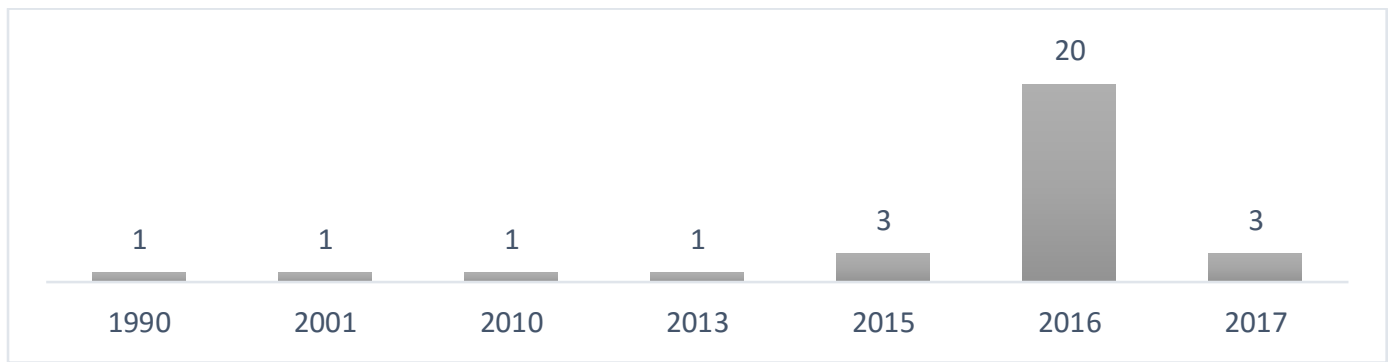

Figure 2: Publication Frequency (1990- 2017)

Until 2015 publications were less frequent, having a couple years where no article was published. Only from this year publications seemed to had a more consistent pattern, with highlights to the year of 2016, reflecting the emergence and recent attention given to the subject. The most cited paper was by Tuominen and Ahlqvist (2010) with 29 citations. Regarding the authors with most publications, a great heterogeneity in the field was observed, each paper analyzed had a different author.

Then, the research makes explicit the predominance of articles in English: 100\% of the publications are in this language. It is observed the relevance of the United States and Germany (Figure 3), tying first in the number of publications.

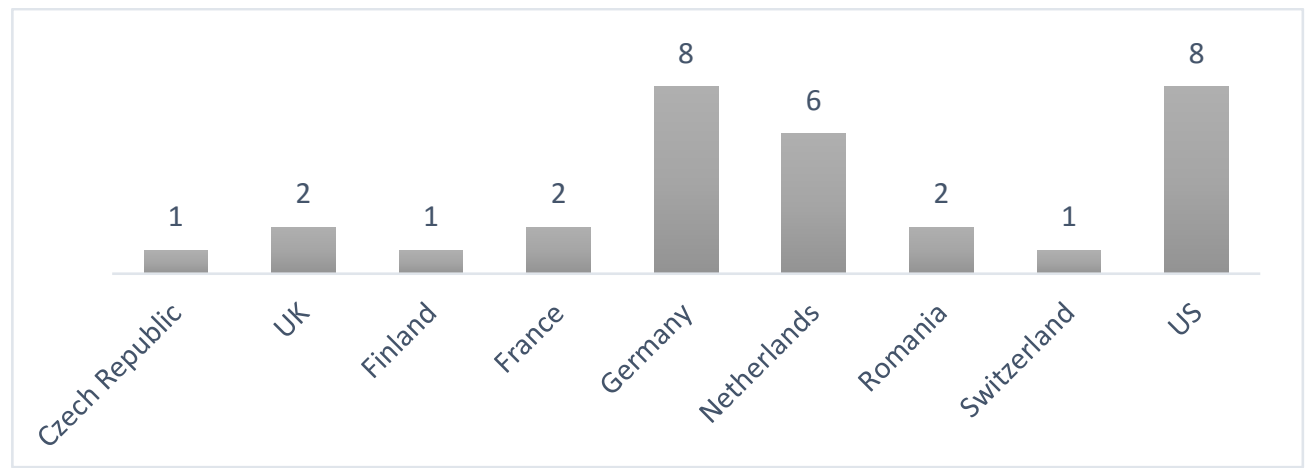

Figure 3: Countries Frequency' Publications.

It is also important to highlight that, by the methodological approach of the papers, we observed that a great majority have a qualitative approach (26 of the 30 articles in total). This fact points to the lack of empirical studies on AVs in the area of M\&B as well as evidencing the emergence of the topic.

Finally, the most cited words in the titles and abstracts of the articles were identified. By using the "Word Frequency Counter" application, Figure 4 presents the most recurrent key-words found in the papers' abstracts and titles. The words were chosen for their frequency and relevance on discussions and debates on the theme. It is important to highlight the words; transport, technology, new, business, model, social, question, system and mobility 


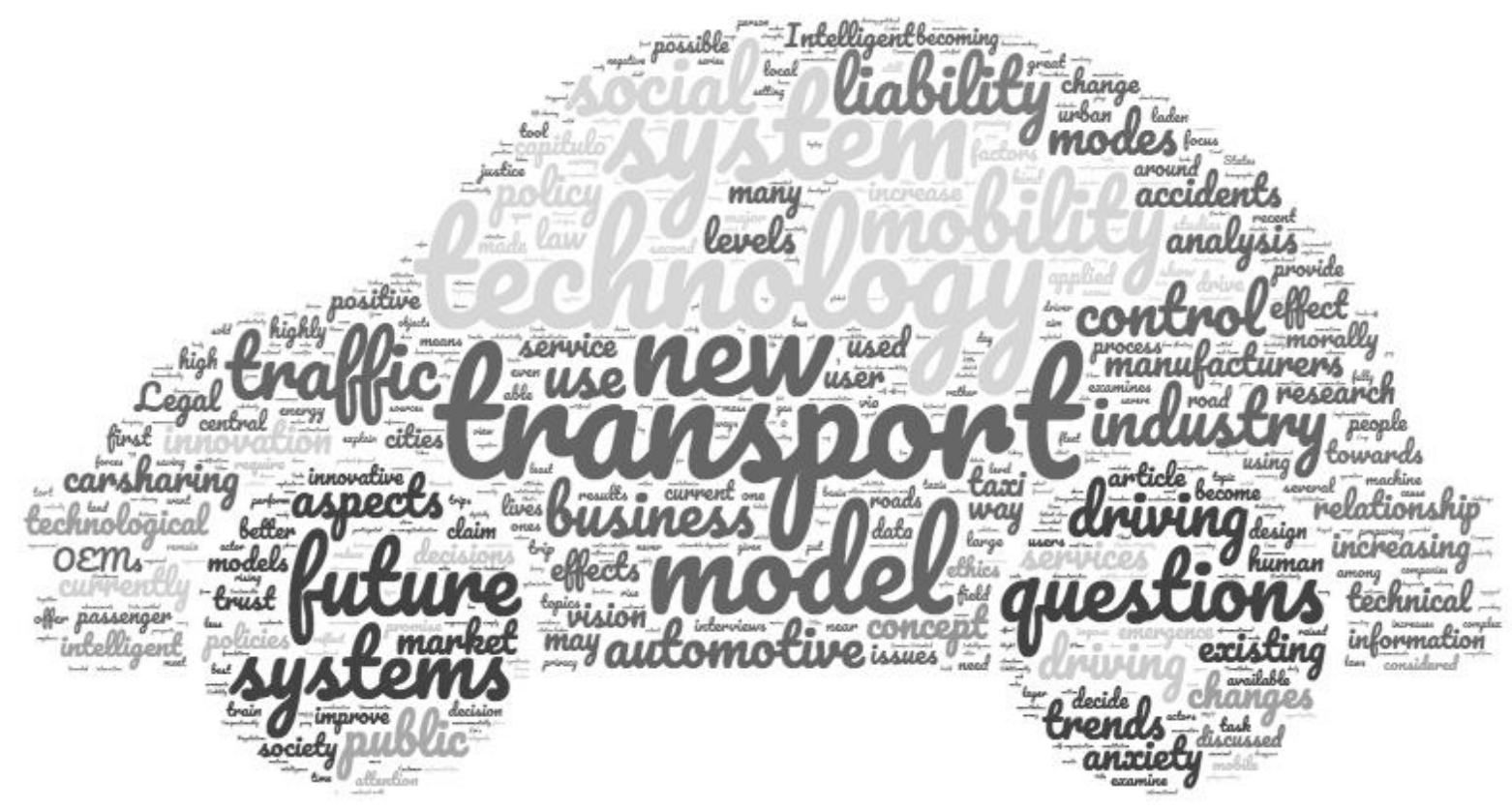

Figure 4: Word cloud of recurrent key-words on the papers' titles and abstracts

\subsection{Integrative review}

In order to carry out the articles' categorization, the analyzes of each of the 30 papers were detailed done, considering subjects related to the research problem, objectives, scope, methodological approach, main contributions, conclusions and recommendations. Regarding the integrative review, a common point observed while creating the synthesis matrix is that there is a consensus among all analyzed papers that AVs will be part of our future society, whether in a short, medium or long-term, "the era of self-driving vehicles is undeniably upon us" (Attias, 2016:p.107)

Uncertainties permeate various aspects of this innovation, being the search for solutions on several questions still unanswered focus of the papers distributed among the evidenced categories. It is also worth noting that there is a strong relationship among all categories, and in many cases, papers identified as belonging to one category broadens their discussions to other categories, leading to the understanding that there are no fixed barriers to the topic.

We also highlight that the main criterion for the inclusion of an article in a given category was the identification of the strongest present theme in its scope. The main themes categorized and detailed in the following sections of this study are: Ethics/Moral/Liability (9 papers); AVs' Business Models (7 papers); Transport Planning (6 papers); Consumer Behavior (4 papers); Policy Issues - Law (4 papers). Although there is a higher incidence of papers in certain categories than in others, we understand that the quantitative metric standard does not infer to a bigger or smaller influence to a conceptual framework formulation for AVs within the field of M\&B. Namely, it is assumed that all the categories identified here are equally necessary, in a synergistic movement, for the formation of the field.

\subsubsection{Ethics/moral/liability}

This category is centered on the necessary and established pre-definitions for the AVs functioning. That is, AVs decision-making, will be previously introduced by someone, "many or all of those decisions will have to be programmed into the car," (Ong, 2017:p.36), therefore, we have the fundamental role of the engineering department, responsible for programming AVs and, consequently, its dissemination. Mladenovic and McPherson (2016) says that decision-making power should not be concentrated solely in a small group of experts, especially if financial interests have exclusive influence. This group should not have exclusive rights to decide on all the moral aspects of these machines behavior (Martin, 2016). In this way, Gogoll \& Müller (2016) affirms that mandatory policies will be more efficient on a large scale, in detriment of include personal ethics settings (in other words, concentrate the decisions of machine behavior in small groups), that is to say, to program all $A V s$ in order not to preserve the life of the occupants in danger from regulations, would be more interesting for all.

One of the biggest challenges for AVs manufacturers is the risk of product liability lawsuits that, in the event of accidents, may result in deaths and catastrophic injuries ( $\mathrm{Wu}, 2016)$. In this way, Hevelke and Nida- 
Rümelin (2015), raise the following question: "who should be made responsible in case of a crash?". The authors conclude that, users of autonomous cars should be collectively responsible for any damage caused by such vehicles-even if they had no way of influencing the cars behavior, and agreed that a tax or a mandatory insurance seems to be the easiest and most practical means to achieve that. Further, Schellekens (2015) says that the chilling effect of product liability on innovation can be further mitigated by adequate obligatory insurance.

Finally, we cannot eliminate the need for a public and democratic discussion of relevant societal values that technology shapes. As a result, it is necessary to engage in a transparent manner all relevant corporate groups in critical discussions and decision-making (Mladenovic \& McPherson, 2016). Change is inescapable and not necessarily a bad thing in itself. But major disruptions and further damage must be anticipated and avoided whenever possible. This is the role of ethics in innovation policy: it can pave the way for a better future, while enabling beneficial technologies. Without looking at ethics, we are driving with one eye closed (Lin, 2016).

\subsubsection{AVs' Business Models}

This category deals with aspects related to the business models proposed for the AVs. The key factors presented in this category refer to the economic implications (including here sale options for these vehicles), as well as discussions about service-oriented business models (e.g. car-sharing), their strengths, opportunities, threats and weaknesses as an option for the deployment of AVs, (Beiker, 2016; Kompalla et al., 2017).

The proposition of business models associated to AVs considering different scenarios of ownership and use - manufacturers, distributors, fleets and public sector is of extreme importance to ensure a good insertion and adherence of AVs in the market. The main gaps in this category point to research that seeks a link between Innovation's theories and approaches to proposing models associated with disruptive innovation, that is, in breaking and redefining existing models so that one can understand and anticipate disruptive business and process strategies and contribute to the construction of a consistent theoretical basis for the new businesses associated with AVs.

\subsubsection{Transport planning}

When it comes to transportation, there is a consensus among the papers of this category that changes will happen and AVs will directly impact or act as accelerators of this process. The development of society is driving these changes, "these developments are being driven by both technology-push and demand-pull factors" (Enoch, 2015:p.922). In view of the digitization context, many industries are gradually being transformed, with AVs as the translation of the digitization of the automotive industry, including the arrival of new players such as Google and Uber (Attias, 2016).

In a gradually changing scenario, Tuominen and Ahlqvist (2010) point to three changes in the future of transport: (1) actor roles and stakeholder networks in the system will be pluralized and the transport system will increasingly be composed of public parties, private entities, taxpayer end-users and complex networks actors; 2) a new type of business and service layer will be formed in the system because of new dynamic interconnections among the actors; 3) a layer of services will be captured from the concept of "technology service", which would be a flexible and adapted combination of technologies and services). Enoch (2015) proposes that these changes will converge from intermediate modes of transport, which will replace traditional transport (cars, buses and taxis). The author believes that the AVs will be accelerated from this process, to a single autonomous universal service of higher quality and cheaper. Attias (2016) proposes that in a mediumterm perspective, this evolution will not stop in the AVs and opens the field for the design of similar technical objects (e.g. trucks, buses) and later boats and autonomous planes.

Finally, Poorsartep (2014) proposes, in addition to a scenario of gradual change, a more radical perspective, which will provide a unique opportunity for the market leader to establish an indisputable position and that can potentially lead to a monopoly.

\subsubsection{Consumer behavior}

The main themes addressed here are questions raised about how it will be and how to measure population and potential consumers' acceptance to AVs. Specially, discussing a) questions of how firms have enhanced trust in applied Artificial Inteligence (Hengstler, Enkel \& Duelli, 2016) , b) examine how positive cognitive evaluations, anxiety-related affects, and the interplay between these two factors influence the willingness to use AVs (Woisetschläger, 2016) c) identify consumer acceptance and branding consequences in the AVs context (Hohenberger, Spörrleb \& Welpe, 2017) and d) present discussions on innovative means to analyze social preferences, demand for AVs, and the potential to resolve community concerns with integrated solutions (Lu et al., 2017). 
When we address issues related to AVs from the consumer's stanpont we clearly perceive that "the main roadblock is human acceptance" (New Scientist, p.5, 2007) We cannot fall into the fallacy of a simple analysis of the benefits that will be brought and/or perceived by the consumer. The feelings of attachment, possession, freedom and the sensations acquired by the consumers as drivers/passengers should be considered.

In fact, rather than presenting solutions and conclusions, these papers introduce preliminary research that opens space for a new theme to be explored when considering the human element, its reactions, preferences and perspectives in the dynamics of AVs as well as the various factors that can influence consumer behavior and willingness to adopt the AVs. The main gaps raised in this category refer to the possibilities of studies that relate different business models (ownership, car-sharing, etc.) to the expectations of different consumers' groups, as well as to compare the cost effectiveness and sustainability of individual ownership versus public-operated AVs. Future studies should also address questions about new forms of time use in vehicles considering the creation of an in-car environment as well as new oportunities of "infoitament" (information + entertainment) that can be offered to consumers by these vehicles. Also, the investigation of individual values and their influence on the adoption of AVs has much to be explored (Hohenberger, Spörrleb \& Welpe, 2017).

\subsubsection{Policy issues - law}

This category presents perspectives based on the lack of clarity about who is responsible for the operation of such vehicles, where this is the major point of confusion among manufacturers, consumers and lawyers. "The liability issue is the biggest one of them all," (Adee, 26, 2016). This lack of clarity may be impacting the way in which public policy has been leading the issues related to the legislation of the AVs. Although there is a consensus in the benefits of AVs, the uncertainties and questions that permeate them are still barriers to the long-range transportation plans (Guerra, 2016).

However, several countries around the world are stimulating, by governmental policies, the dissemination of the AVs. Autonomous driving is not just about the automobile industry but about many other industrial branches that will benefit from a greater degree of automation such as component suppliers. That is why there is a growing interest in promoting local advantages and why political commitments are beginning to be made in some countries to support certain paths of development (Schreurs and Steuwer, 2016).

In this sense, this study approaches considered that policies issues will act in two stages: 1) to stimulate companies and investments in order to provide technological advances and consequent dissemination of the AVs (current stage of the AVs); 2) development of public policies, or adequacy of laws for AVs. It is understood that this stage will also follow local fators, which would allow an assessment of how the corresponding legal framework should be designed to make a real "road traffic system" involving autonomous vehicles (Gasser, 2016).

\subsection{Discussion}

Figure 5 illustrates the categories developed in this study as well as points to the main issues related to each of them.

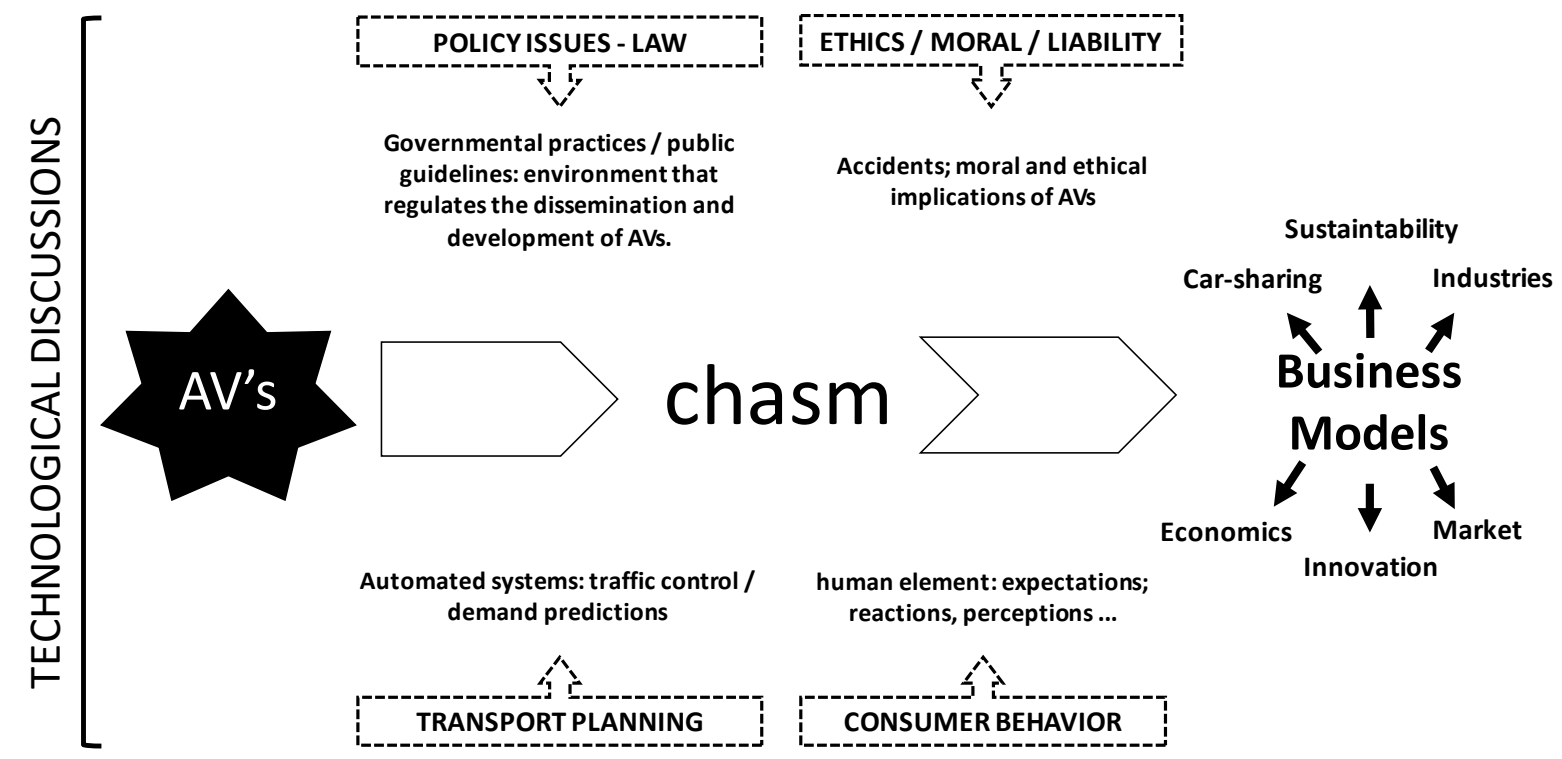

Figure 5: Theoretical framework of AVs M\&B 
As shown on Figure 06, several aspects and macro-environments permeate the AVs. Currently the vast majority of research related to such vehicles, tend to focus on more technical (technological) aspects related to the vehicle's characteristics itself. The thematic categories drawn in this study point to central trends of studies related to other knowledge areas raising important questions on the perspectives and implications of the AVs arrival. Analyzing the framework one can observe that, until AVs can be implemented and actually "cross the chasm" to mainstream there are several legal (policy); ethical and moral issues as well as discussion regarding transportation planning and consumer behavior that need to be addressed. Finally, the business model category points to the need to think about and elaborate new models and market standards for AVs.

\section{Concluding remarks}

AVs stand out as a technological innovation that will translate the yearnings of a new era into our society. The field's evolution reflects in a broad conceptual framework regarding AVs (the first search on the subject yielded more than 35,000 papers), however when refining the search for M\&B field of study fewer works were yielded, indicating a possible gap between the technological evolution of this innovation and its eventual disruption, considering that the business models plays an extremely important role in the events that precede advancement of AVs (Yun et al., 2016), so that the way in which this innovation might be established by uncertainties, may impact directly on governments' lack of planning for such arrival (Guerra, 2016).

In order to reduce these uncertainties, this work identified five knowledge categories, separated by a thin line, implying in a synergistic orientation among them, which contributes to the field's disruptive appeal. It was observed that businesses models should be consumer oriented, however these should be aligned with society's ethical and moral precepts, as well as with government policies for future transport planning. Namely, the automotive industry must understand the need to establish technological advances that do not neglect social impacts over profitability. In the opposite direction, entities opposed to this advance should keep in mind that such technological evolution is inevitable and they must make efforts to reconcile this advance, which must be added of governmental support to balance the equation.

Finally, it is understood that many uncertainties permeate the theme, and this study sought to identify the academic efforts to foster contributions that could result in a favorable advancement of the field. From this, it is understood that the joint evolution of the categories that permeate the field of the AVs in M\&B will be determinant for a positive innovation result that will configure the future of our society in detriment of a disorderly advance that can cause irreparable damages.

\section{Acknowledgments}

This work would not have been possible without the citation records of scientific publications from Thomson Reuters' Web of Science and CiteSpace software. We also would like to thank the Post Graduate Program in Administration of the Federal University of Lavras, the Terrestrial Mobility Laboratory (LMT-UFLA), the Laboratoire Génie Industriel (LGI - CentraleSupélec), especially the research group of Sustainable Economy and the funding institutions of this project, Coordenação de Aperfeiçoamento de Pessoal de Nível Superior (CAPES) and Fundação de Amparo à Pesquisa de Minas Gerais (Fapemig).

\section{References}

Adee, S. (2016) Who is in control. New Scientist.

Attias, D. (2016). The Autonomous Car, a Disruptive Business Model?. In The Automobile Revolution (pp. 99113). Springer International Publishing.

Chen, K., \& Ervin, R. D. (1990). Intelligent vehicle-highway systems: US activities and policy issues. Technological Forecasting and Social Change, 38(4), 363-374.

Christensen, C. M., Baumann, H., Ruggles, R., \& Sadtler, T. M. (2006). Disruptive innovation for social change. Harvard business review, 84(12), 94.

Directorate-General for Internal Policie. (2016). Research for TRAN Committee - Self-piloted cars: The future of road transport? Accessed in January, 20 th of 2017, from http://www.europarl.europa.eu/RegData/etudes/STUD/2016/573434/IPOL_STU(2016)573434_EN.pdf

Editorial. (2007). No driver required. What's to stop us sitting back and letting the car drive itself? New Scientist. Enoch, M. P. (2015). How a rapid modal convergence into a universal automated taxi service could be the future for local passenger transport. Technology Analysis \& Strategic Management, 27(8), 910-924.

Fagnant, D. J., Kockelman, K. (2015). Preparing a nation for autonomous vehicles: opportunities, barriers and policy recommendations. Trasponrtation Research Part A: Policy and Practice, 77, 167-181. 
Frazzoli, E., Dahleh, M. A., Feron, E. (2002). Real-time motion planning for agile autonomous vehicles. Journal of Guidance, Control, and Dynamics, 25(1), 116-129.

Gasser, T. M. (2016). Fundamental and Special Legal Questions for Autonomous Vehicles. In Autonomous Driving (pp. 523-551). Springer Berlin Heidelberg.

Gogoll, J., \& Müller, J. F. (2016). Autonomous cars: in favor of a mandatory ethics setting. Science and Engineering Ethics, 1-20.

Guerra, E. (2016). Planning for cars that drive themselves: Metropolitan Planning Organizations, regional transportation plans, and autonomous vehicles. Journal of Planning Education and Research, 36(2), 210224.

Hengstler, M., Enkel, E., \& Duelli, S. (2016). Applied artificial intelligence and trust-The case of autonomous vehicles and medical assistance devices. Technological Forecasting and Social Change, 105, 105-120.

Hevelke, A., \& Nida-Rümelin, J. (2015). Responsibility for crashes of autonomous vehicles: an ethical analysis. Science and engineering ethics, 21(3), 619-630.

Hohenberger, C., Spörrle, M., \& Welpe, I. M. (2017). Not fearless, but self-enhanced: The effects of anxiety on the willingness to use autonomous cars depend on individual levels of self-enhancement. Technological Forecasting and Social Change, 116, 40-52.

Lima, D. A. (2015). Sensor-based navigation applied to intelligent electric vehicles. Doctoral thesis, Université De Technologie De Compiègne, Compiègne, France.

Lin, P. (2016). Why ethics matters for autonomous cars. In Autonomous Driving (pp. 69-85). Springer Berlin Heidelberg.

Lu, Z., Du, R., Dunham-Jones, E., Park, H., \& Crittenden, J. (2017). Data-enabled public preferences inform integration of autonomous vehicles with transit-oriented development in Atlanta. Cities, 63, 118-127.

Martin, D. (2016). Who Should Decide How Machines Make Morally Laden Decisions?. Science and Engineering Ethics, 1-17.

Mladenovic, M. N., \& McPherson, T. (2016). Engineering social justice into traffic control for self-driving vehicles?. Science and engineering ethics, 22(4), 1131-1149.

Mutz, F., Veronese, L. P., Oliveira-Santos, T., de Aguiar, E., Cheein, F. A. A., \& De Souza, A. F. (2016). Large-scale mapping in complex field scenarios using an autonomous car. Expert Systems with Applications, 46, 439462.

Ong, S. (2017). Auto correct. New Scientist

Poorsartep, M. (2014). Self-Driving Cars: Radical Innovation in the Transportation Industry. In Transcultural Marketing for Incremental and Radical Innovation (pp. 96-105). IGI Global.

Schellekens, M. (2015). Self-driving cars and the chilling effect of liability law. Computer Law \& Security Review, 31(4), 506-517.

Schoitsch, E. (2016). Autonomous Vehicles and Automated Driving Status, Perspectives and Societal Impact. Information Technology, Society and Economy Strategic Cross-Influences (IDIMT-2016). 24th Interdisciplinary Information Management Talks, 45(1), 405-424.

Schreurs, M. A., \& Steuwer, S. D. (2016). Autonomous Driving-Political, Legal, Social, and Sustainability Dimensions. In Autonomous Driving (pp. 149-171). Springer Berlin Heidelberg.

Tuominen, A., \& Ahlqvist, T. (2010). Is the transport system becoming ubiquitous? Socio-technical roadmapping as a tool for integrating the development of transport policies and intelligent transport systems and services in Finland. Technological forecasting and social change, 77(1), 120-134.

Woisetschläger, D. M. (2016). Consumer Perceptions of Automated Driving Technologies: An Examination of Use Cases and Branding Strategies. In Autonomous Driving (pp. 687-706). Springer Berlin Heidelberg.

Wu, S. S. (2016). Product Liability Issues in the US and Associated Risk Management. In Autonomous Driving (pp. 553-569). Springer Berlin Heidelberg.

Yun, J. J., Won, D., Jeong, E., Park, K., Yang, J., \& Park, J. (2016). The relationship between technology, business model, and market in autonomous car and intelligent robot industries. Technological Forecasting and Social Change, 103, 142-155. 